\section{Dialogue entre cellules \\ endothéliales \\ et cellules \\ musculaires lisses}

\author{
Michel Félétou, Rudi Busse, Gillian Edwards, Ingrid \\ Fleming, Arthur H. Weston, \\ Paul M. Vanhoutte
}

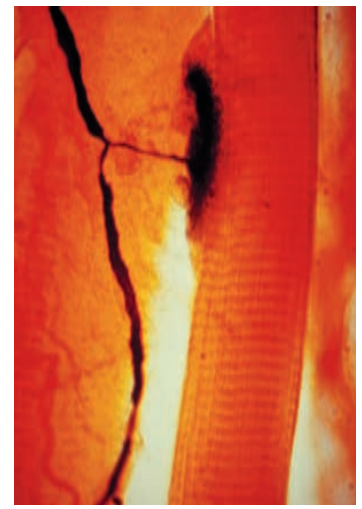

\author{
M. Félétou, P.M. Vanhoutte : \\ Institut de Recherches Servier, \\ 92150 Suresnes, France. \\ R. Busse, I. Fleming, \\ A.H. Weston : \\ Institut für Kardiovaskuläre \\ Physiologie, Klinikum der \\ J.W. Goethe-Universität, \\ Frankfurt, Allemagne. \\ G. Edwards : School of Biological \\ Sciences, University of \\ Manchester, Manchester M13 9PT, \\ Royaume-Uni. \\ michel.feletou@fr.netgrs.com
}

Les cellules endothé-

liales synthétisent et sécrètent plusieurs substances vasodilatatrices ou vasoconstrictrices en réponse à des stimulus biochimiques (peptides, neuroamines...) ou à des stimulus physiques (force de cisaillement, pression). Au cours des 25 dernières années, deux vasodilatateurs d'origine endothéliale ont été identifiés et caractérisés, la prostacycline synthétisée par la cyclooxygénase à partir de l'acide arachidonique, et le monoxyde d'azote (NO) produit par la NO-synthase à partir de la L-arginine [1]. Cependant, la production de prostacycline ou de $\mathrm{NO}$ ne peut expliquer l'ensemble des relaxations (ou vasodilatations) dépendantes de l'endothélium. En présence d'inhibiteurs de cyclo-oxygénase et de $\mathrm{NO}$-synthase, un mécanisme additionnel responsable d'une vasorelaxation, toujours dépendante de l'endothélium, est observé dans de nombreuses artères de différentes espèces, y compris chez l'homme (Figure 1). Ce mécanisme implique l'hyperpolarisation des cellules musculaires lisses et il est particulièrement prépondérant dans la circulation coronaire et dans les lits vasculaires périphériques [2].

Calcium et canaux potassiques endothéliaux 
La stimulation des récepteurs endothéliaux de l'acétylcholine, de la bradykinine ou de la substance P provoque une augmentation du calcium intracellulaire $\left([\mathrm{Ca}]_{\mathrm{i}}\right)$ due à la fois à une libération de calcium des stocks internes et à une entrée de calcium externe grâce à l'ouverture de conductances cationiques (les cellules endothéliales n'exprimant pas les canaux calciques dépendants du potentiel). Les relaxations dépendantes de l'endothélium produites par la prostacycline ou le NO sont étroitement corrélées aux variations de $[\mathrm{Ca}]_{\mathrm{i}}$ de la cellule endothéliale [3]. II en est de même pour les hyperpolarisations, dépendantes de l'endothélium, résistantes aux inhibiteurs de cyclo-oxygénase et de NO-synthase [4]. Ces hyperpolarisations sont inhibées par une augmentation de la concentration de potassium extracellulaire et par des inhibiteurs des canaux potassiques. Il a donc tout d'abord été suggéré que ces hyperpolarisations dépendantes de l'endothélium impliquaient un troisième facteur également sécrété par les cellules endothéliales en réponse à une élévation de $[\mathrm{Ca}]_{\mathrm{i}}$. Ce facteur non identifié appelé $\varepsilon D H F$ (endothelium-derived hyperpolarizing factor) était

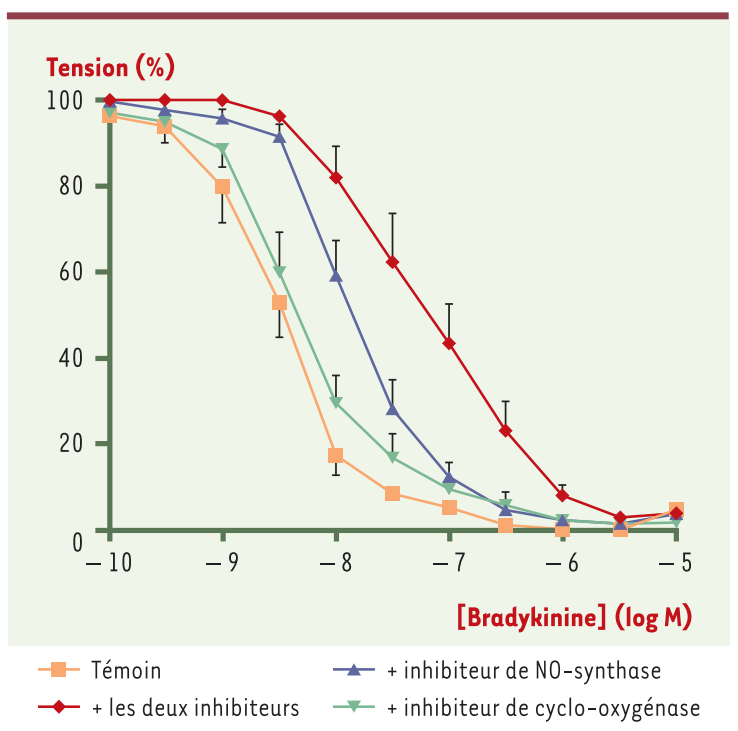

Figure 1. Courbes de concentration-relaxation en réponse à la bradykinine sur un anneau d'artère coronaire isolée de chien. La bradykinine produit une relaxation dépendante de l'endothélium qui n'est que partiellement inhibée par la présence conjointe d'un inhibiteur de cyclo-oxygénase (indométacine) et de NO-synthase (L-nitro-arginine). La composante de la relaxation qui subsiste en présence de ces deux inhibiteurs est attribuée à un mécanisme additionnel qui implique l'hyperpolarisation des cellules musculaires lisses (EDHF, endotheliumderived hyperpolarizing factor). supposé diffuser vers les cellules musculaires lisses sous-jacentes et activer leurs canaux potassiques [5]. Cependant, cette hypothèse a été battue en brèche par de récentes observations.

\section{EDHF et canaux potassiques activés par le calcium}

Les réponses attribuées à l'EDHF sont bloquées de façon spécifique par l'association de deux toxines provenant de venins de scorpion et d'abeille, respectivement la charybdotoxine et l'apamine [6, 7] (Figure 2). L'apamine bloque spécifiquement un canal potassique de faible conductance dépendant du calcium $\left(\mathrm{SK}_{\mathrm{ca}}\right)$, alors que la charybdotoxine est un inhibiteur moins spécifique qui inhibe différents canaux potassiques, y compris des canaux dépendants du calcium de grande conductance $\left(\mathrm{BK}_{\mathrm{Ca}}\right)$ et de conductance intermédiaire $\left(I K_{C a}\right)$. Or une autre toxine de scorpion, l'ibériotoxine, inhibiteur spécifique des $B K_{\text {ca }}$, ne reproduit pas, dans la plupart des études in vitro, l'effet de la charybdotoxine $[8,9]$, indiquant que les $B K_{c a}$ ne sont certainement pas impliqués dans la majorité des réponses attribuées à l'EDHF.

Localisation des canaux potassiques $\mathrm{SK}_{\mathrm{Ca}}$ et $\mathrm{IK}_{\mathrm{Ca}}$ : les cellules endothéliales

Les cellules musculaires lisses vasculaires, dans leur phénotype contractile, expriment les $B K_{C a}$, mais elles expriment peu ou pas les $\mathrm{SK}_{\mathrm{Ca}}$ ou les $I \mathrm{~K}_{\mathrm{Ca}}$. À l'inverse, des cellules endothéliales fraîchement dissociées expriment les $S K_{C a}$ et les $I K_{C a}$, mais peu ou pas les BK $K_{c a}[10-$ 12]. Ces observations suggèrent que les cibles des deux toxines sont situées sur les cellules endothéliales et non sur les cellules musculaires lisses. Cette hypothèse a été étayée par plusieurs observations. L'activation de récepteurs endothéliaux produit une augmentation de $[\mathrm{Ca}]_{\mathrm{i}}$ dans les cellules endothéliales, qui elle-même entraîne l'activation des canaux $I K_{c a}$ et $S K_{c a}$ sensibles, respectivement, à la charybdotoxine et l'apamine (Figure 3). Il en résulte une hyperpolarisation de la cellule endothéliale, qui est bloquée par la combinaison de ces deux toxines [11-14]. Le décours temporel de I'hyperpolarisation des cellules endothéliales et des cellules musculaires lisses est identique [15]. Dans un vaisseau perfusé, le traitement associant les deux toxines est efficace si l'administration est effectuée dans la lumière du vaisseau (les toxines sont en contact avec les cellules endothéliales), mais demeure inefficace si l'administration est effectuée dans le liquide baignant l'extérieur du vaisseau (les toxines sont en contact avec la face adventitielle du vaisseau et ont donc pour cible prioritaire les cellules musculaires lisses) [16]. Au total, l'activation des canaux 
endothéliaux $\mathrm{SK}_{\mathrm{Ca}}$ et IK $\mathrm{K}_{\mathrm{Ca}}$ et, en conséquence, I'hyperpolarisation des cellules endothéliales sont requises pour observer une hyperpolarisation des cellules musculaires lisses.

\section{Transmission de l'hyperpolarisation endothéliale vers les cellules musculaires lisses}

Pour rendre compte de la transmission de l'hyperpolarisation endothéliale vers les cellules musculaires lisses, plusieurs hypothèses ont été évoquées (pour revue, voir [5]). Les principales font appel à la transmission au travers de jonctions de type communicantes (gap junctions), à la fuite et l'accumulation de potassium dans l'espace intercellulaire, et à la production d'acides époxyeicosatriénoïques (દદT), métabolites instables de l'acide arachidonique produits par une cytochrome P450 mono-oxygénase. inhibent les réponses attribuées à l'EDHF [20].

\section{Les ions potassium}

L'hyperpolarisation des cellules endothéliales - grâce à l'ouverture des conductances potassiques - entraîne de fait une fuite de potassium de l'espace intracellulaire vers l'espace extracellulaire. Le potassium peut s'accumuler dans l'espace intercellulaire, entre les cellules endothéliales et les cellules musculaires lisses. Or une augmentation modérée de la concentration en potassium extracellulaire (2-15 mM) peut provoquer l'hyperpolarisation des cellules musculaires lisses en mettant en jeu deux mécanismes différents, l'activation d'un canal potassique rectifiant dans le sens entrant $\left(K_{\mathrm{IR}}\right)$ et/ou l'activation de certaines isoformes de la pompe $\mathrm{Na}^{+}, \mathrm{K}^{+}$-ATPase (voir Encadré). Dans ce cas, l'ion $\mathrm{K}^{+}$ représente le facteur $\varepsilon D H F$. Ce concept a été établi et sa pertinence vérifiée en mesurant le potentiel de mem-

\section{Les gap junctions}

Les cellules endothéliales, ainsi que les cellules musculaires lisses, sont couplées entre elles par des gap junctions, impliquant diverses connexines telles les connexines 37,40 et 43 . II existe également des liaisons hétérocellulaires myoendothéliales, couplant les cellules endothéliales aux cellules musculaires lisses. Le nombre de ces jonctions myoendothéliales augmente au fur et à mesure que le diamètre artériel diminue, une évolution qui est parallèle à l'augmentation de la contribution de la composante EDHF dans les relaxations dépendantes de l'endothélium. De plus, dans des vaisseaux dont les relaxations dépendantes de l'endothélium ne comportent pas de composante $\varepsilon D H F$, comme l'artère fémorale de rat, les jonctions myoendothéliales sont absentes [17]. Les cellules endothéliales et musculaires lisses peuvent communiquer par ces jonctions puisque le calcium peut diffuser d'une cellule à l'autre [18]. La conduction d'une dépolarisation ou d'une hyperpolarisation d'une cellule musculaire lisse vers une cellule endothéliale (ou à l'inverse d'une cellule endothéliale vers une cellule musculaire lisse) a été démontrée dans plusieurs artères de faible diamètre, montrant que, dans ces vaisseaux, les cellules endothéliales et musculaires lisses peuvent former un véritable syncytium [19-22]. Dans certaines artères, des inhibiteurs de gap junctions

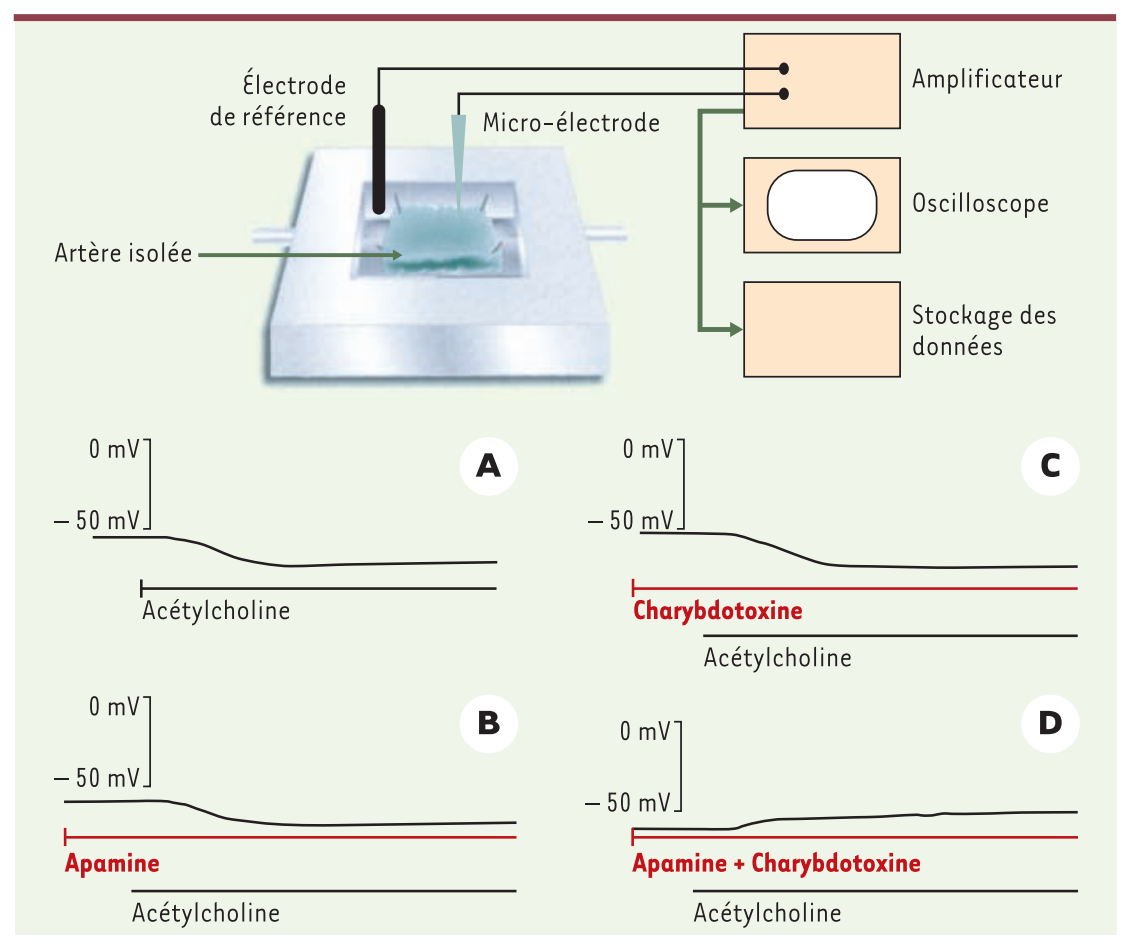

Figure 2. Cellules musculaires lisses: enregistrement du potentiel de membrane et effet d'inhibiteurs des canaux potassiques. Le potentiel de membrane est enregistré dans une cellule musculaire lisse d'un segment d'artère carotide de cobaye pourvue de son endothélium. L'acétylcholine produit une hyperpolarisation de la cellule musculaire lisse si et seulement si l'endothélium est présent (A). Cette hyperpolarisation persiste en présence d'un inhibiteur de cyclo-oxygénase (indométacine) et de NO-synthase (L-nitro-arginine) (non montré sur cette figure). L'apamine (B), un inhibiteur spécifique d'un canal potassique dépendant du calcium de faible conductance $\left(S K_{C a}\right)$, et la charybdotoxine $(C)$, un inhibiteur moins spécifique de différents canaux potassiques, y compris des canaux de conductance intermédiaire dépendants du calcium ( $\left.K_{\text {Ca }}\right)$, n'ont aucun effet lorsqu'ils sont administrés seuls. Cependant la combinaison de ces deux toxines (D) abolit l'hyperpolarisation dépendante de l'endothélium (d'après [7]). 
brane des cellules endothéliales et des cellules musculaires lisses d'artères hépatique et mésentérique de rat [14]. Cependant, I'implication des ions $\mathrm{K}^{+}$dans les réponses attribuées à l'EDHF n'a pu être démontrée dans tous les vaisseaux sanguins étudiés.

\section{Les dérivés de la cytochrome P450 mono-oxygénase}

À partir d'expériences réalisées principalement sur des gros troncs coronaires d'origine canine, porcine ou bovine, de nombreux auteurs ont suggéré que les réponses attribuées à l'EDHF impliquaient les acides époxyeicosa-

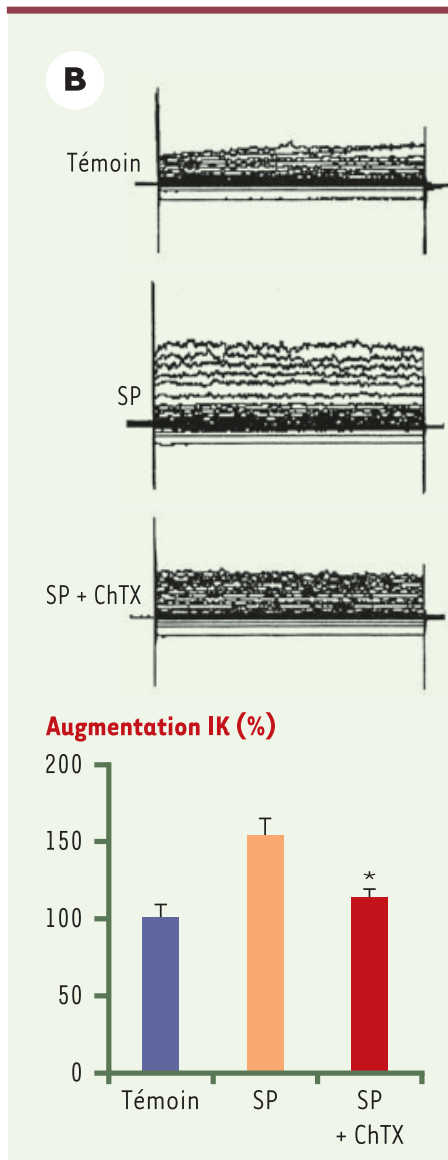

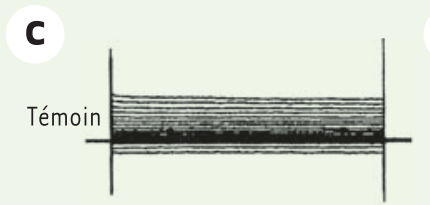

A
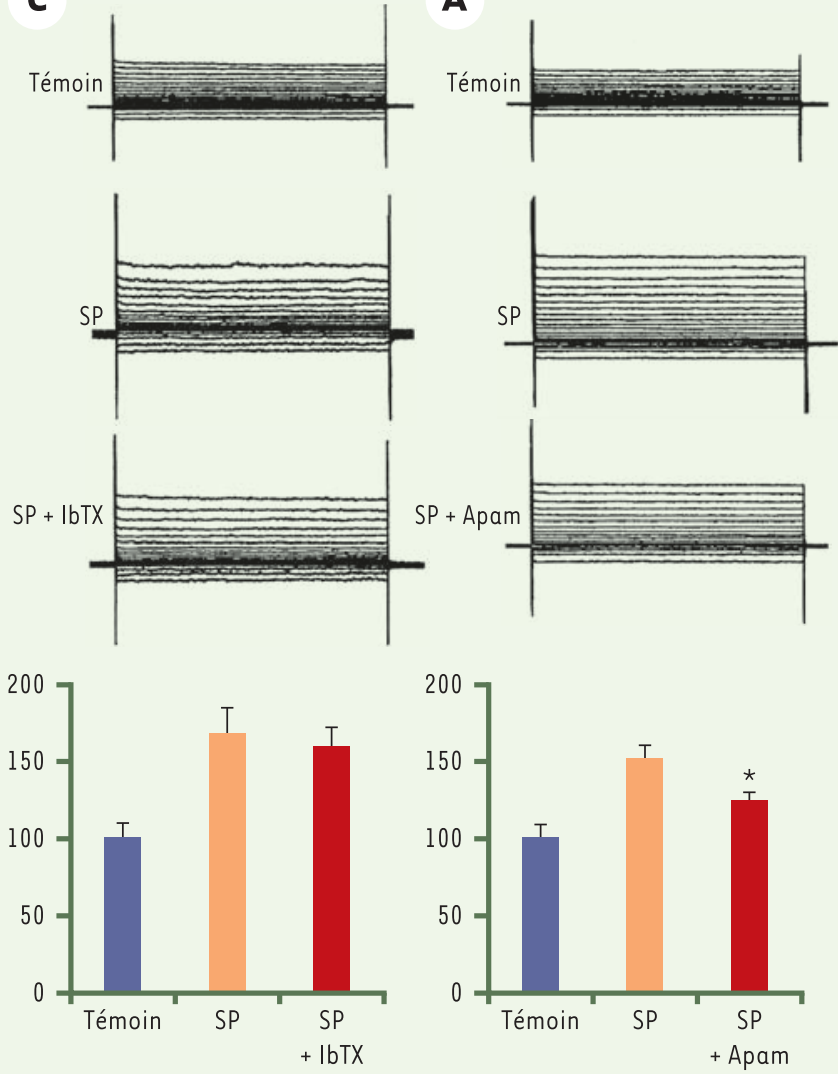

triénoïques (દદT) [23, 24]. Les દદT relaxent de nombreuses artères et hyperpolarisent les cellules musculaires lisses des artères coronaires en activant des $\mathrm{BK}_{\mathrm{Ca}}$. Les réponses attribuées à l'EDHF, obtenues sur ces gros troncs coronaires, sont fréquemment réduites, voire abolies, par des inhibiteurs de cytochrome P450 ou par des oligonucléotides antisens dirigés contre une isoforme particulière de la cytochrome P450 mono-oxygénase, exprimée par les cellules endothéliales, la P450 2C8-9 [25]. Enfin, un antagoniste des દદT récemment synthétisé (14,15-દદZદ, acide 14,15 époxyéicosa-5Z-énoïque) inhibe non seulement les réponses produites par l'administration d'દદT, mais également les réponses attribuées à l'EDHF [26]. Ces travaux suggèrent donc que, dans les gros troncs coronaires, l'EDHF est un દET qui, après synthèse et sécrétion par les cellules endothéliales, aurait pour cible la cellule musculaire lisse et entraînerait son hyperpolarisation en activant une conductance potassique. Cependant, même si une sécrétion d'EદT est mesurée à partir de cellules endothéliales bovines, elle est plus difficile à mettre en évidence à partir d'autres cellules ou de segments artériels isolés. De plus, comme cela a déjà été mentionné cidessus, la majorité des réponses attribuées à l'EDHF ne met pas en jeu les canaux $\mathrm{BK}_{\mathrm{Ca}}$. Enfin, si les દદT peuvent exercer une action sur les cellules musculaires lisses, ces composés jouent également un rôle majeur au sein de la cellule endothéliale [27].

\section{Une hypothèse \\ fédératrice}

Les hyperpolarisations dépendantes de l'endothélium ne proviennent pas nécessairement d'une multitude de réponses différentes variant au gré de 
l'espèce, du tissu ou des conditions expérimentales. Un modèle fédérateur (Figure 4) a pu être proposé [2]. L'événement premier et commun correspond à l'augmentation de $[\mathrm{Ca}]_{\mathrm{i}}$ au sein des cellules endothéliales, suivi par l'activation des conductances potassiques et par l'hyperpolarisation de ces cellules. Dans les gros troncs coronaires, cette réponse endothéliale est sous la dépendance de l'activité de la cytochrome P450 monooxygénase; les દદT sont des messagers intracellulaires endothéliaux pouvant régler l'homéostasie calcique [28], les conductances potassiques [29] et la perméabilité des gap junctions [30]. La libération dans le milieu extracellulaire de ces દદT ne doit pas être exclue, mais elle ne se produirait que dans certaines circonstances, par exemple en réponse à un stimulus agressif [31]. L'hyperpolarisation de la cellule endothéliale peut se propager de manière électrotonique via les gap junctions. Cependant, l'ouverture des canaux potassiques endothé-

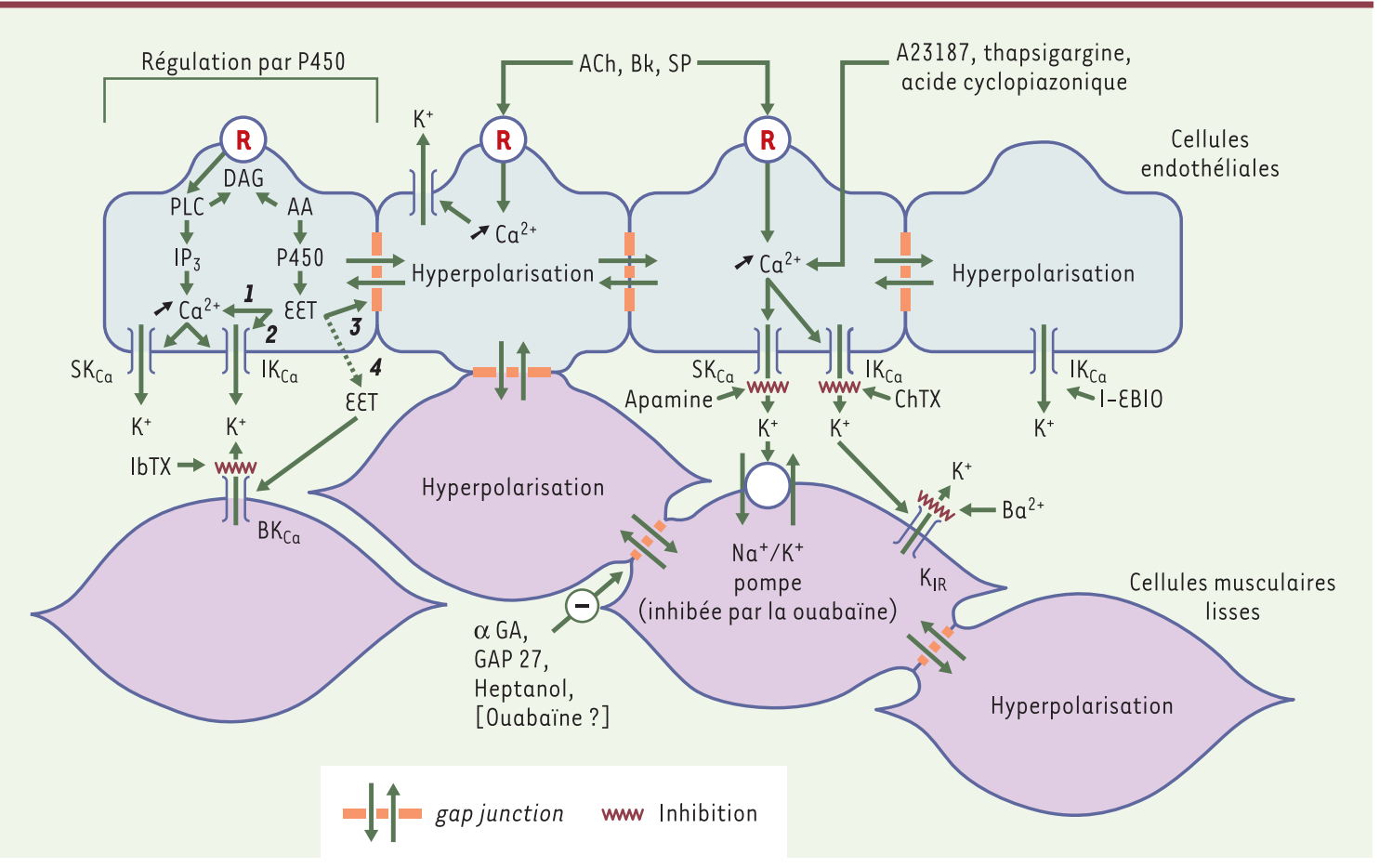

Figure 4. Une hypothèse fédératrice pour expliquer les hyperpolarisations dépendantes de l'endothélium. Dans les hyperpolarisations dépendantes de l'endothélium, l'événement premier est l'augmentation du $[\mathrm{Ca}]_{\mathrm{i}}$ au sein des cellules endothéliales, suivi de l'activation des conductances potassiques endothéliales $\left(S K_{\mathrm{Ca}}\right.$ et $\left.\mathrm{IK}_{\mathrm{Ca}}\right)$ et de l'hyperpolarisation de la cellule endothéliale. Cette hyperpolarisation peut se propager vers les cellules musculaires lisses de manière électrotonique via les jonctions communicantes myoendothéliales. Cependant, l'ouverture des canaux potassiques endothéliaux a pour conséquence immédiate la fuite de potassium et l'accumulation de celui-ci dans l'espace intercellulaire, phénomène qui peut également hyperpolariser les cellules musculaires lisses en activant une conductance potassique rectifiant dans le sens entrant $\left(K_{I R}\right)$ et/ou certaines isoformes de la pompe $\mathrm{Na}^{+}, \mathrm{K}^{+}$-ATPase. Dans les gros troncs coronaires, la réponse endothéliale est sous la dépendance de la cytochrome P450 mono-oxygénase. Dans ces conditions, les acides époxyeicosatriénoïques (દદT) sont des messagers intracellulaires endothéliaux pouvant contrôler la libération de calcium des stocks intracellulaires (1), les conductances potassiques (2) et la perméabilité des gap junctions (3). La libération dans le milieu extracellulaire de ces દદT ne doit pas être exclue (4) et, lorsqu'elle se produit, les દદT activent des canaux $B_{C a}$ situés sur les cellules musculaires lisses. A23187: ionophore calcique; AA: acide arachidonique; ACh: acétylcholine; BK: bradykinine; DAG: diacyl-glycérol; દદT: acide époxyeicosatriénoïque; $\mid P_{3}$ : inositol trisphosphate; P450: cytochrome P450 mono-oxygénase; PLC: phospholipase C; R: récepteur; SP: substance P. L'ibériotoxine (IbTX) est un inhibiteur spécifique des $B K_{C a}$. La charybdotoxine (ChTX) est un inhibiteur des $B K_{C a}$, des $I K_{C a}$ et de certains canaux potassiques dépendants du potentiel. L'apamine est un inhibiteur spécifique des SK $K_{C a}$. L'l-éthyl-2-benzimidazolinone (1-EBIO) ouvre les canaux IK $K_{C a}$. L'ouabaïne, à des concentrations submicromolaires, est un inhibiteur de la $\mathrm{Na}^{+}, \mathrm{K}^{+}-A T P a s e$. Le baryum $\left(\mathrm{Ba}^{2+}\right)$, à des concentrations millimolaires, est un inhibiteur relativement spécifique des canaux $K_{\mathbb{R}}$. Le Gap27, un peptide de 11 acides aminés possédant une homologie de séquence avec une portion de la seconde boucle extracellulaire d'une connexine, le $18 \alpha$-glycyrrhetinic acid ( $\alpha G A)$, ainsi que l'heptanol sont des inhibiteurs de gap junctions (d'après [44]). 
liaux a pour conséquence immédiate la fuite de potassium et l'accumulation de celui-ci dans l'espace intercellulaire, phénomène qui peut également hyperpolariser les cellules musculaires lisses. Ces divers mécanismes ne sont pas nécessairement exclusifs, ils peuvent se produire simultanément, être additifs, voire synergiques. La prépondérance de l'un ou l'autre de ces mécanismes dans les réponses attribuées à l'EDHF dépendra du degré d'expression des protéines impliquées et des caractéristiques intrinsèques de ces protéines. Par exemple, pour que l'accumulation de potassium dans le milieu extracellulaire puisse hyperpolariser le muscle lisse vasculaire sous-jacent, il est nécessaire que les canaux $K_{\mathbb{R}}$ et/ou que les isoformes appropriées de la pompe $\mathrm{Na}^{+}, \mathrm{K}^{+}$-ATPase

A

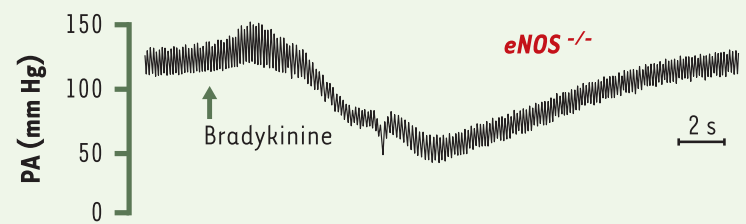

B

B
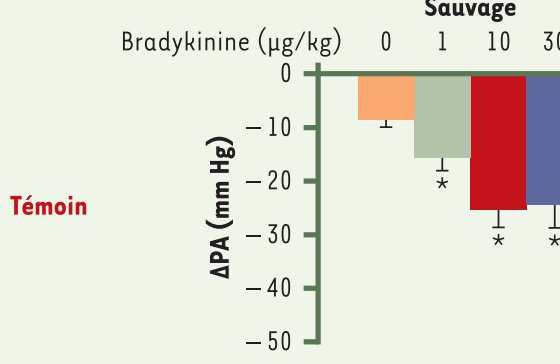

Sauvage Bradykinine $(\mu \mathrm{g} / \mathrm{kg}) \quad 0 \quad \begin{array}{ccc}\text { Sauvage } & 10 & 30\end{array}$

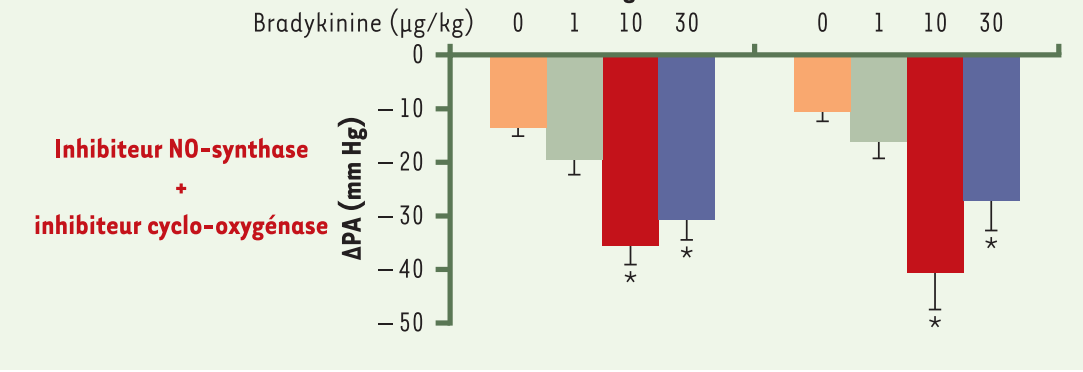

Figure 5. Pression artérielle chez des souris anesthésiées déficientes pour la forme endothéliale de la NO-synthase [eNOS${ }^{-/}$] et les souris sauvages (C57BL6). A. Tracé original montrant les effets d'une administration intraveineuse en bolus de bradykinine $(10 \mu \mathrm{g} / \mathrm{kg})$ sur la pression artérielle (PA) d'une souris eNOS ${ }^{-/}$préalablement traitée avec un inhibiteur de cyclo-oxygénase, le diclofénac (10 mg/kg, i.p.) et un inhibiteur de NO-synthase, l'ester méthylé de la Lnitro-arginine (L-NAME, $30 \mathrm{mg} / \mathrm{kg}$, i.p.). B. Effet de l'administration, sous forme de bolus, de sérum physiologique (colonne orange) ou de bradykinine ( 1,10 et $30 \mu \mathrm{g} / \mathrm{kg}$, respectivement pour les colonnes vertes, rouges et bleues) sur la pression artérielle moyenne en l'absence (témoin), ou en présence de diclofénac et de L-NAME. La chute de pression provoquée par l'administration de bradykinine n'est pas différente entre les souris eNOS ${ }^{-1-}$ traitées et celles non traitées par des inhibiteurs de cyclo-oxygénase de $\mathrm{NO}$-synthase, ce qui montre que la voie de I'EDHF joue un rôle majeur dans le contrôle local du débit sanguin (d'après [38]). soient présents et fonctionnels dans ces cellules muscu-

\section{Les réponses attribuées à l'EDHF :}

Les hyperpolarisations dépendantes de l'endothélium sont associées à des relaxations du muscle lisse vascucium et, d'autre part, la diminution de la production de phosphatidylinositol intracellulaire qui réduira la libération de calcium des sites de stockage intracellulaire [35]. Dans les vaisseaux sanguins de différentes espèces, y compris chez l'homme, parmi les mécanismes impliqués dans les relaxations dépendantes de l'endothélium, la contribution de l'EDHF devient de plus en plus prépondérante au fur et à mesure que le diamètre artériel décroît $[36,37]$. Chez la souris, les vasodilatations attribuées à l'EDHF sont au moins aussi importantes que celles produites par le NO endothélial, puisque ni la délétion du gène de la $\mathrm{NO}$-synthase endothéliale, ni l'inhibition des $\mathrm{NO}$-synthases n'atténuent l'hypotension (Figure 5) ou les vasodilatations produites par l'administration d'acétylcholine ou de bradykinine [38]. Chez le rat anesthésié, I'application locale de la combinaison des deux toxines, charybdotoxine et apamine, ne produit pas d'effet sur les résistances basales de divers lits vasculaires périphériques (mésentérique et train arrière), mais inhibe partiellement l'augmentation de conductance produite par l'acétylcholine [39]. Toutes ces données indiquent que, si l'EDHF joue un rôle modeste dans la régulation des conductance basales, il contribue à la régulation locale des résistances périphériques vasculaires et donc à la répartition du flux sanguin en réponse à divers messagers neurohumoraux.

Des altérations de la voie de l'EDHF ont été montrées tant chez l'homme que chez l'animal. Ainsi, le vieillissement (Figure 6), l'hypercholestérolémie, l'hypertension artérielle essentielle ainsi que 
l'éclampsie gravidique diminuent les réponses attribuées à l'EDHF dans les artères humaines. De plus, ces réponses sont également altérées chez l'animal dans divers modèles d'hypertension, de diabète, d'athérosclérose ou de choc septique [37, 40-44]. À l'inverse, les réponses attribuées à l'EDHF peuvent exercer un rôle compensateur quand la voie de la $\mathrm{NO}$-synthase est altérée chez les patients hypertendus [45] ou dans des modèles animaux de dénudation et de régénération endothéliale [46].

L'amélioration des réponses attribuées à l'EDHF peut contribuer à la restauration de la fonction endothéliale lors de différentes interventions thérapeutiques. Les inhibiteurs de l'enzyme de conversion de l'angiotensine (Figure 6), les antagonistes des récepteurs ATl de l'angiotensine ou les œstrogènes augmentent les hyperpolarisations dépendantes de l'endothélium. II est intéressant de noter que cet effet des œstrogènes a été associé chez le rat à une augmentation de l'expression de la connexine 43, qui est exprimée par les cellules endothéliales et musculaires lisses, suggérant une implication des gap junctions, et potentiellement une augmentation des jonctions myoendothéliales dans l'effet bénéfique des œstrogènes [41-44].

\section{Conclusions}

Le mécanisme des hyperpolarisations dépendantes de l'endothélium commence à être mieux compris. Des toxines ont été identifiées et utilisées pour bloquer de façon sélective cette voie. Ces outils sont cependant encore imparfaits et ne permettent pas de façon aisée une exploration fonctionnelle chez l'animal, ni bien entendu chez l'homme. Cependant, plus nos connaissances progressent et plus il apparaît que les réponses attribuées à l'EDHF jouent un rôle physiologique majeur dans le contrôle local du débit sanguin. $\diamond$

\section{SUMMARY}

EDHF : a new pathway for endothelial and smooth muscle cells communication Vascular endothelial cells play a fundamental role in the control of vascular tone, and therefore in the control of local blood flow, by releasing various contracting (endothelin, prostaglandins) and relaxing (prostacycline, NO) factors. An additional mechanism involving the hyperpolarization of the vascular smooth muscle cells is observed mainly in the coronary vascular bed and in the periphery. This phenomenon was attributed to an elusive endothelial factor called endothelium-derived hyperpolarizing factor (EDHF). This mechanism is now better understood. It involves first an increase in the endothelial intracellular concentration of calcium, the activation of endothelial potassium channels and the resulting hyperpolarization of the endothelial cells. The hyperpolarization of the endothelial cells is transmitted to the smooth muscle cells by different pathways. This hyperpolarization propagates along the vessels not only via the smooth muscle cells but also via the endothelial cells. Therefore, the endothelial layer can also be considered as a conducting tissue. The discovery of specific inhibitors of the endothelial cell hyperpolarization allows the assessment of the contribution of EDHF-mediated responses in the control of vascular tone. $\diamond$

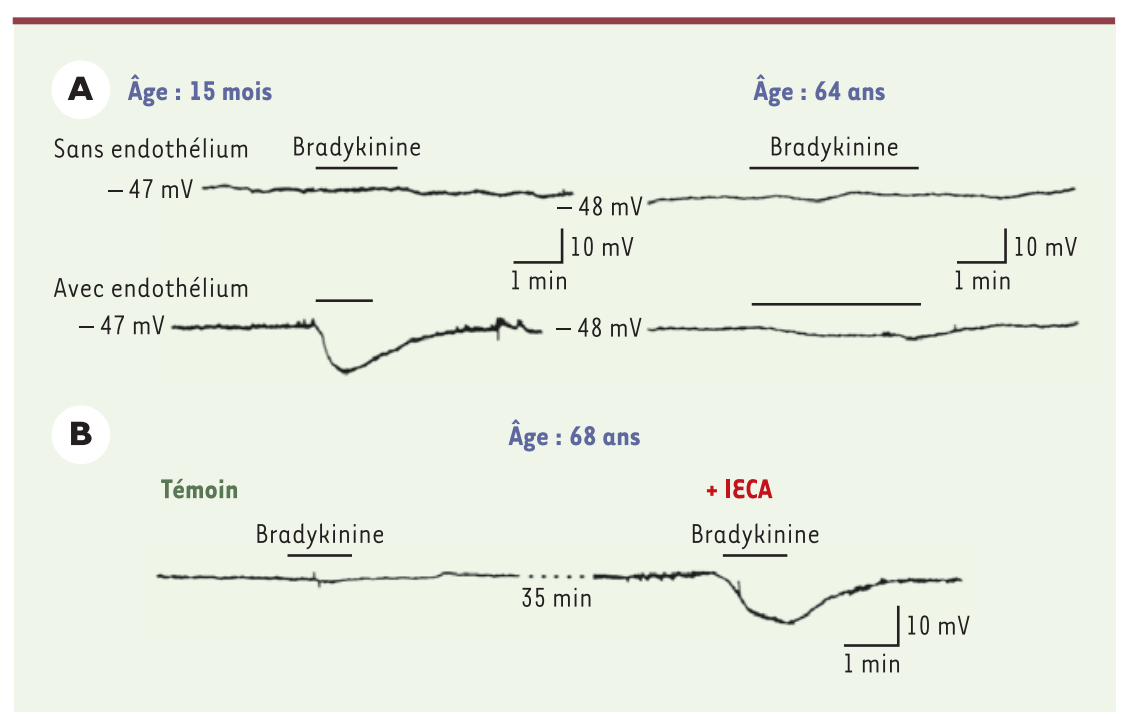

Figure 6. Effet de l'âge, des pathologies ischémiques myocardiques ainsi que de l'inhibition de l'enzyme de conversion de l'angiotensine sur les hyperpolarisations dépendantes de l'endothélium de l'artère coronaire humaine. Le potentiel de membrane des cellules musculaires lisses de segments d'artères coronaires humaines isolées, pourvues ou non de leur endothélium, a été enregistré à l'aide de microélectrodes intracellulaires. Les expériences ont été réalisées en présence des inhibiteurs de cyclo-oxygénase (indométacine, $10^{-5} \mathrm{M}$ ) et de $\mathrm{NO}$-synthase (L-nitro-arginine, $10^{-4} \mathrm{M}$ ). A. Les enregistrements ont été réalisés sur des artères coronaires de deux patients a priori non porteurs de maladies coronariennes et âgées respectivement de 15 mois et de 64 ans. L'application de bradykinine $\left(10^{-7} \mathrm{M}\right)$ produit une hyperpolarisation des cellules musculaires lisses qui dépend de l'endothélium et qui est nettement diminuée dans l'artère coronaire du sujet âgé. $B$. Les enregistrements ont été obtenus sur un même segment d'artère coronaire provenant d'un patient souffrant d'ischémie myocardique âgé de 68 ans. Alors que l'application de bradykinine $\left(10^{-8} \mathrm{M}\right)$ ne produit aucune hyperpolarisation dépendante de l'endothélium (témoin), une préincubation de $30 \mathrm{~min}$ avec du périndoprilate $\left(10^{-6} \mathrm{M}\right)$, un inhibiteur de l'enzyme de conversion de l'angiotensine (IECA), restaure une hyperpolarisation dépendante de l'endothélium en réponse à une seconde application de bradykinine (d'après $[40,42]$ ). 


\section{ACCUMULATION DE POTASSIUM हT HYPERPOLARISATION}

- Il pourrait paraître illogique qu'une augmentation de la concentration extracellulaire de potassium puisse provoquer l'hyperpolarisation des cellules musculaires lisses et/ou endothéliales si l'on suit la règle de la loi de Nernst.

- En fait, la pompe $\mathrm{Na}^{+}, \mathrm{K}^{+}$-ATPase est activée par le potassium extracellulaire. $\mathrm{La} \mathrm{Na}^{+}, \mathrm{K}^{+}$-ATPase étant électrogénique, son activation produit une hyperpolarisation. Les différentes isoformes de la $\mathrm{Na}^{+}, \mathrm{K}^{+}$-ATPase exprimées dans le muscle lisse (ou l'endothélium) n'ont pas la même sensibilité vis-à-vis du potassium externe. L'isoforme contenant la sous-unité $\alpha_{1}$, exprimée de façon ubiquitaire, est généralement complètement activée pour des concentrations physiologiques de potassium $(\sim 5 \mathrm{mM})$. En revanche, les isoformes contenant les sous-unités $\alpha_{2}$ ou $\alpha_{3}$, (exprimées dans le muscle lisse de l'artère mésentérique de rat, un vaisseau où la réponse EDHF implique le potassium, [32]) sont activées pour des concentration de potassium plus élevées (jusqu'à 15-20 mM).

- Une augmentation de la concentration en potassium extracellulaire peut également conduire à une hyperpolarisation par l'activation du canal potassique rectifiant dans le sens entrant $\left(K_{\mathbb{R}}\right)$. Ce canal, activé par le potassium extracellulaire, a la particularité de présenter une courbe courant-potentiel en forme de N. Ce courant n'est sortant que dans une fenêtre étroite de potentiel, située entre environ $-50 \mathrm{mV}$ (le potentiel de repos pour les cellules musculaires lisses et endothéliales) et $-20 \mathrm{mV}$. Lorsque ce canal est activé, dans cette fenêtre de potentiel, il produit une hyperpolarisation de la cellule considérée. Ce canal potassique est exprimé par les cellules endothéliales et les cellules musculaires lisses des petites artères de résistance (diamètre < $300 \mu \mathrm{m}$ ) [14, 33-35].

- L'activation de la pompe $\mathrm{Na}^{+}, \mathrm{K}^{+}$-ATPase et/ou de $\mathrm{K}_{\mathrm{R}}$, par une augmentation modérée de la concentration en potassium extracellulaire, compense largement la faible dépolarisation que l'on pourrait attendre en suivant l'équation de Nernst.
3. Luckhoff A, Pohl U, Mulsch A, Busse R. Differential role of extra- and intracellular calcium in the release of EDRF and prostacyclin from cultured endothelial cells. Br J Pharmacol 1988 ; 95: 189-96.

4. Ghisdal P, Morel N. Cellular target of voltage and calcium-dependent $\mathrm{K}^{+}$ channel blockers involved in EDHF-mediated responses in rat superior mesenteric artery. $\mathrm{Br} J$

Pharmacol 2001 ; 134 :1021-8.

5. McGuire JJ, Ding H, Triggle CR. Endothelium-derived relaxing factors: a focus on endothelium-derived hyperpolarizing factor. Can J Physiol Pharmacol $2001 ; 79: 443-70$.

6. Garland CJ, Plane F. Relative importance of endothelium-derived hyperpolarizing factor for the relaxation of vascular smooth muscle in different arterial beds. In: Vanhoutte PM, ed. Endotheliumderived hyperpolarizing factor, vol. 1. Amsterdam: Harwood Academic Publishers, 1996: 173-9.

7. Corriu C, Félétou M, Canet $\varepsilon$, Vanhoutte PM. Endothelium-derived factors and hyperpolarisations of the isolated carotid artery of the guinea-pig. $\mathrm{Br} J$ Pharmacol 1996 ; $119: 959-64$.

8. Chataigneau T, Félétou M, Duhault J, Vanhoutte PM. Epoxyeicosatrienoic acids, potassium channel blockers and endotheliumdependent hyperpolarisation in the guinea-pig carotid artery. BrJ Pharmacol 1998 ; $123: 574-80$.

9. Zygmunt PM, Hogestatt $\varepsilon D$. Role of potassium channels in endothelium-dependent relaxation resistant to nitroarginine in the rat hepatic artery. $\mathrm{Br}$ J Pharmacol 1996 ; 117 : 1600-6.
10. Quignard JF, Félétou M, Edwards G, Duhault J, Weston AH, Vanhoutte PM. Role of endothelial cells hyperpolarization in EDHFmediated responses in the guinea-pig carotid artery. BrJ Pharmacol 2000 ; 129 : 1103-12.

11.Burnham MP, Bychkov R, Félétou $M$, et al. Characterization of an apamin-sensitive small conductance $\mathrm{Ca}^{2+}$ activated $\mathrm{K}^{+}$channel in porcine coronary artery endothelium: relevance to EDHF. BrJ Pharmacol 2002 ; $135: 1133-43$

12. Bychkov R, Burnham MP, Richards GR, et al. Characterization of a charybdotoxin-sensitive intermediate conductance $\mathrm{Ca}^{2+}$-activated $\mathrm{K}^{+}$channel in porcine coronary endothelium: relevance to EDHF. BrJ Pharmacol 2002 ; 137 : 1346-54.

13. Busse R, Fichtner $H$, Luckhoff A, Kohlhardt M. Hyperpolarisation and increased free calcium in acetylcholine-stimulated endothelial cells. Am J Physiol 1988 ; 255: H965-9.

14.Edwards G, Dora KA, Gardener MJ, Garland CJ, Weston $\mathrm{AH} . \mathrm{K}^{+}$is an endothelium-derived hyperpolarizing factor in rat arteries. Nature 1998 ; $396: 269-72$.

15.Beny JL. Endothelial and smooth muscle cells hyperpolarized by bradykinin are not dye coupled. Am J Physiol 1990 ; 258 : H836-4l.

16. Doughty JM, Plane F, Langton PD. Charybdotoxin and apamin block EDHF in rat mesenteric artery if selectively applied to the endothelium. Am J Physiol 1999 ; 276 : H1107-12.

17. Sandow SL, Tare M, Coleman HA, Hill CVE, Parkington HC. Involvement of gap junctions in the action of endothelium-derived hyperpolarizing factor. Circ Res 2002 ; $90: 1108-13$. 
18.Dora KA, Doyle MP, Duling BR. Elevation of intracellular calcium in smooth muscle causes endothelial cell generation of NO in arterioles. Proc Natl Acad Sci USA 1997 ; $94: 6529-34$.

19. Beny JL, Pacicca C Bidirectional electrical communication between smooth muscle and endothelial cells in the pig coronary artery. Am J Physiol 1994 ; 266: Hl465-72.

20.Chaytor AY, Evens WH, Griffith TM. Central role of heterocellular gap junction communication in endothelium-dependent relaxations of rabbit arteries. J Physiol (London) 1998 ; 508 : 561-73.

21. Yamamoto $Y$, Imaeda K, Suzuki H. Endotheliumdependent hyperpolarization and intercellular electrical coupling in guinea-pig mesenteric arterioles.J Physiol (London) 1999 ; 514 : 505-13.

22.Emerson GG, Segal SS. Electrical coupling between endothelial cells and smooth muscle cells in hamster feed arteries: role in vasomotor control. Circ Res $2000 ; 87: 474-9$

23. Campbell WB, Gebremedhin D, Pratt PF, Harder DR. Identification of epoxyeicosatrienoic acids as endothelium-derived hyperpolarizing factor. Circ Res 1996; $78: 415-23$.

24. Quilley J, McGiff JC. Is EDHF an epoxyeicosatrienoic acid? Trends Pharmacol Sci $2000 ; 21: 121-4$

25. Fisslthaler B, Popp R, Kiss L, et al. Cytochrome P450 2C is an EDHF synthase in coronary arteries. Nature 1999 ; 401 : 493-7.

26. Gauthier KM, Deeter C, Krishna UM, et al. 14,15epoxyeicosa-5(Z)-enoic acid: a selective epoxyeicosatrienoic acid antagonist that inhibits endothelium-dependent hyperpolarization and relaxation in coronary arteries. Circ Res 2002 ; 90 : 1028-36.
27. Fleming I, Fisslthaler $B$, Michaelis UR, Kiss L, Popp $R$, Busse R. The coronary EDHF stimulates multiple signalling pathways and proliferation in vascular cells. Pfluger's Arch Eur J Physiol 2001 ; 442 : 511-8.

28. Hoebel BG, Kostner GM, Graier WF. Activation of microsomal P450 monooxygenase by $\mathrm{Ca}^{2+}$ store depletion and its contribution to $\mathrm{Ca}^{2+}$ entry in porcine aortic endothelial cells. Br J Pharmacol 1997 ; 121 : 1579-88

29. Baron A, Frieden M, Bény JL. Epoxyeicosatrienoic acids activate a highconductance, $\mathrm{Ca}^{2+}$ dependent $\mathrm{K}^{+}$channel on pig coronary artery endothelial cells. J Physiol (London) 1997 ; $504: 537-43$

30.Popp R, Brandes RP, Ott G, Busse R, Fleming I. Dynamic modulation of interendothelial gap junctional communication by 11,12 epoxyeicosatrienoic acid. Circ Res 2002 ; 90 : 800-6.

31.Popp R, Fleming I, Busse R. Pulsatile stretch in coronary arteries elicits release of endotheliumderived hyperpolarizing factor: a modulator of arterial compliance. Cir Res $1998 ; 82$ : 696-703

32. Weston $A H$, Richards GR, Burnham MP, Félétou $M$, Vanhoutte PM, Edwards G. $\mathrm{K}^{+}$-induced hyperpolarization in rat mesenteric artery: identification, localization and role of $\mathrm{Na}^{+}, \mathrm{K}^{+}$-ATPases. BrJ Pharmacol 2002 ; 136: 918-26.

33. Quignard JF, Félétou M, Thollon C, Vilaine JP, Duhault J, Vanhoutte PM. Potassium ions and endothelium-derived hyperpolarizing factor in guinea-pig carotid and porcine coronary arteries. BrJ Pharmacol 1999 ; $127: 27-34$.

34.Richards GR, Burnham MP,
Edwards G, Félétou $M$, Vanhoutte PM, Weston AH. Suppression of $\mathrm{K}^{+}$-induced hyperpolarization by phenylephrine in rat mesenteric artery: relevance to studies of endothelium-derived hyperpolarizing factor. $\mathrm{Br} J$ Pharmacol 2001 ; 134 : 1-5.

35. Nelson MT, Patlak JB, Worley JF, Standen NB. Calcium channels, potassium channels, and voltage dependence of arterial smooth muscle tone. Am J Physiol 1990 ; 259 : C3-18.

36. Shimokawa $H$, Yasutake $H$, Fujii $K$, et al. The importance of the hyperpolarizing mechanism increases as the vessel size decrease in endotheliumdependent relaxations in rat mesenteric circulation. J Cardiovasc Pharmaco 1996 ; 28 : 703-11.

37. Urakami-Harasawa L, Shimokawa $\mathrm{H}$, Nakashima M, Egashira K, Takeshita A. Importance of endothelium-derived hyperpolarizing factor in human arteries. J Clin Invest 1997 ; $100: 2793-9$.

38. Brandes RP, SchmitzWinnenthal FH, Félétou $M$, et al. An endothelium-derived hyperpolarizing factor distinct from NO and prostacyclin is a major endothelium-dependent vasodilator in resistance vessels of wild type and endothelial NO synthase knock-out mice. Proc Natl Acad Sci USA 2000 . 57 : 9747-52.

39. Parkington HC, Chow JA, Evans RG, Coleman HA, Tare M. Role for endotheliumderived hyperpolarizing factor in rat mesenteric and hindlimb circulation in vivo. J Physiol (London) 2002 ; 542: 929-37.

40. Nakashima M, Mombouli JV,
Taylor AA, Vanhoutte PM. Endothelium-dependent hyperpolarisation caused by bradykinin in human coronary arteries. J Clin Invest 1993 ; 92 : 2867-71.

41.Vanhoutte PM. Endotheliumderived hyperpolarizing factor. Amsterdam: Harwood Academic Publishers, 1996 : $338 \mathrm{p}$.

42. Vanhoutte PM. Endotheliumdependent hyperpolarizations. Amsterdam: Harwood Academic Publishers, 1999 : 436 p.

43. Vanhoutte PM. EDHF 2000. London: Taylor and Francis, 2001: 502 p.

44.Vanhoutte PM. EDHF 2002. London: Taylor and Francis, 2004 (sous presse).

45.Taddei $S$, Ghiadoni L, Virdis A, Buralli S, Salvetti A. Vasodilatation to bradykinin is mediated by an ouabain-sensitive pathway as a compensatory mechanism for impaired nitric oxide availability inessential hypertensive patients. Circulation 1999 ; $100: 1400-5$

46. Thollon C, Bidouard JP, Cambarrat $C$, et al. Alteration of endotheliumdependent hyperpolarizations in porcine coronary arteries with regenerated endothelium. Circ Res 1999 ; 84 : 371-7. 